\title{
General solution of Bernoulli and Riccati fractional differential equations based on conformable fractional derivative
}

\author{
M. Ilie ${ }^{1,2}$, J. Biazar ${ }^{1,3}$, Z. Ayati ${ }^{4, *}$ \\ ${ }^{1}$ Department of Mathematics, Guilan Science and Research Branch, Islamic Azad University, Rasht, Iran \\ ${ }^{2}$ Department of Mathematics, Rasht Branch, Islamic Azad University, Rasht, Iran \\ ${ }^{3}$ Department of Applied Mathematics, Faculty of Mathematical Sciences, University of Guilan,P.O.Box.41938, Rasht, Iran \\ ${ }^{4}$ Department of Engineering sciences, Faculty of Technology and Engineering East of Guilan, \\ University of Guilan, P.C. 44891-63157, Rudsar-Vajargah, Iran \\ *Corresponding author E-mail: ayati.zainab@gmail.com
}

\begin{abstract}
This paper aimed to develop two well-known nonlinear ordinary different equations, Bernoulli and Riccati equations to fractional form. General solution to fractional differential equations are detected, based on conformable fractional derivative. For each equation, numerical examples are presented to illustrate the proposed approach.
\end{abstract}

Keywords: Fractional Differential Equations; Conformable Fractional Derivative; Bernoulli Fractional Differential Equation; Riccati Fractional Differential Equation.

\section{Introduction}

Solving fractional different equations is very important but there are many fractional different equations which can't be solved analytically. Due to this fact, finding an approximate solution of fractional different equations is clearly an important task. In recent years, many effective methods have been proposed for the approximate solution to classical fractional differential equations, such as Adomian decomposition method [3], [4], homotopy perturbation method [5-7], homotopy analysis method [8], variational iteration method [9], generalized differential transform method [10], finite difference method [11], Laplace transform method [12], Sub-equation method [13], Legendre operational matrix [14], etc.

The organization of this paper is as follows: In Section 2, the basic ideas conformable fractional derivative, and conformable fractional integral are described. In Section 3, Bernoulli and Riccati fractional differential equations are solved. Finally, conclusion and discussions are presented in section 4 .

\section{Basic definitions}

The purpose of this section is to recall some results on the proposed method.

\subsection{Conformable fractional derivative}

Given a function $\mathrm{f}:[0, \infty) \rightarrow \mathbb{R}$. Then conformable fractional derivative of $f$ of order $\alpha$ is defined by

$$
T_{\alpha}(f)(x)=\lim _{\varepsilon \rightarrow 0} \frac{f\left(x+\varepsilon x^{1-\alpha}\right)-f(x)}{\varepsilon}
$$

For all $\mathrm{x}>0, \alpha \in(0,1]$. If $\mathrm{f}$ is $\alpha$ - differentiable in some $(0, \mathrm{a}), \mathrm{a}>$ 0 , and $\lim _{\mathrm{x} \rightarrow 0^{+}} \mathrm{T}_{\alpha}(\mathrm{f})(\mathrm{x})$ exists, then one can define

$T_{\alpha}(f)(0)=\lim _{x \rightarrow 0^{+}} T_{\alpha}(f)(x)$

If the conformable derivative of $\mathrm{f}$ of order $\alpha$ exists, then we simply say that $\mathrm{f}$ is $\alpha$-differentiable (see $[1,2]$ ).

One can easily show that $T_{\alpha}$ satisfies all the following properties (see [1]):

Let $\alpha \in(0,1]$ and be $\alpha$-differentiable at a point $\mathrm{x}>0$, Then

A. For $a, b \in \mathbb{R} T_{\alpha}(a f+b g)=a T_{\alpha}(f)+b T_{\alpha}(g)$,

B. For all $\mathrm{p} \in \mathbb{R} \mathrm{T}_{\alpha}\left(\mathrm{x}^{\mathrm{p}}\right)=\mathrm{px}^{\mathrm{p}-\alpha}$,

C. For all constant functions $\mathrm{f}(\mathrm{x})=\lambda, \mathrm{T}_{\alpha}(\lambda)=0$,

D. $T_{\alpha}(f \cdot g)=g \cdot T_{\alpha}(f)+f \cdot T_{\alpha}(g)$,

E. $T_{\alpha}\left(\frac{f}{g}\right)=\frac{g \cdot T_{\alpha}(f)-f \cdot T_{\alpha}(g)}{g^{2}}$,

F. $T_{\alpha}(f)=x^{1-\alpha} \frac{d f}{d x}$.

\subsection{Conformable fractional integral}

Given a function $\mathrm{f}:[\mathrm{a}, \infty) \rightarrow \mathbb{R}, \mathrm{a} \geq 0$. Then conformable fractional integral of $f$ is defined by

$I_{\alpha}^{a}(f)(x)=\int_{a}^{x} \frac{f(t)}{x^{1-\alpha}} d x$

Where the integral is the usual Riemann improper integral, and $\alpha \in$ $(0,1)$ (see [1], [2]). 
For simplicity, we have, $\mathrm{I}_{\alpha}^{0}(\mathrm{f})(\mathrm{x})=\mathrm{I}_{\alpha}(\mathrm{f})(\mathrm{x})$

One of the well results is the following (see [1]):

For all $\mathrm{x} \geq \mathrm{a}$, and any continuous function in the domain of $\mathrm{I}_{\alpha}^{\mathrm{a}}$, we have $\mathrm{T}_{\alpha}\left(\mathrm{I}_{\alpha}^{\mathrm{a}} \mathrm{f}(\mathrm{x})\right)=\mathrm{f}(\mathrm{x})$.

\section{Basic theoretical of the idea}

Bernoulli and Riccati equations will be converted in to a first order linear equation, by changing dependent variable. In this section, the procedure of the solution of first order linear fractional equations is described. Then general solution to Bernoulli and Riccati fractional equations are presented.

\subsection{First order linear fractional differential equations}

The most important type of fractional different equations is the linear fractional differential equation, in which the conformable fractional derivative of highest order is a linear function of the lower conformable fractional derivative. Thus, the general first order fractional differential equation based on conformable fractional derivative is presented as

$$
\mathrm{T}_{\alpha}(\mathrm{u})(\mathrm{x})=\mathrm{p}(\mathrm{x}) \mathrm{u}(\mathrm{x})+\mathrm{q}(\mathrm{x}),
$$

Where $\mathrm{p}(\mathrm{x}), \mathrm{q}(\mathrm{x})$ are $\alpha$-differentiable functions and $\mathrm{u}(\mathrm{x})$ is an unknown function.

We write in the standard form,

$\mathrm{T}_{\alpha}(\mathrm{u})(\mathrm{x})+\mathrm{P}(\mathrm{x}) \mathrm{u}(\mathrm{x})=\mathrm{Q}(\mathrm{x})$

By using the property $(\mathrm{F})$, equation (4) will be written as

$\mathrm{x}^{1-\alpha} \mathrm{u}^{\prime}(\mathrm{x})+\mathrm{P}(\mathrm{x}) \mathrm{u}(\mathrm{x})=\mathrm{Q}(\mathrm{x})$

$u^{\prime}(x)+\frac{P(x)}{x^{1-\alpha}} u(x)=\frac{Q(x)}{x^{1-\alpha}}$.

Where equation (5) is a first order linear ordinary differential equation with general solution

$u(x)=e^{-\int \frac{P(x)}{x^{1-\alpha}} d x}\left(\int \frac{Q(x)}{x^{1-\alpha}} e^{\int \frac{P(x)}{x^{1-\alpha}} d x} d x+C\right)$,

Wherever $\mathrm{C}$ is constant among and arbitrary (see [15]). Now by using subsection (2.2) and substitution in equation (6) we obtain

$\mathrm{u}(\mathrm{x})=\mathrm{e}^{-\mathrm{I}_{\alpha}(\mathrm{P}(\mathrm{x}))}\left[\mathrm{I}_{\alpha}\left(\mathrm{Q}(\mathrm{x}) \cdot \mathrm{e}^{\mathrm{I}_{\alpha}(\mathrm{P}(\mathrm{x}))}\right)+\mathrm{C}\right]$.

As a results (7) is the general solution to equation (4).

Example 1 According to equation (7) the following first order linear fractional differential equation

$\mathrm{T}_{\frac{1}{2}}(\mathrm{u})(\mathrm{x})+\mathrm{u}(\mathrm{x})=\mathrm{x}^{2}+2 \mathrm{x}^{\frac{3}{2}}$

Has a general solution such as,

$u(x)=x^{2}+C e^{-2 \sqrt{x}}$.

Example 2 According to equation (7) a general solution of the following first order linear fractional differential equation

$\mathrm{T}_{\frac{1}{2}}(\mathrm{u})(\mathrm{x})+\sqrt{\mathrm{x}} \mathrm{u}(\mathrm{x})=\mathrm{xe}^{-\mathrm{x}}$

Is as the following

$\mathrm{u}(\mathrm{x})=\mathrm{e}^{-\mathrm{x}}\left(\frac{2}{3} \mathrm{x} \sqrt{\mathrm{x}}+\mathrm{C}\right)$

\subsection{Bernoulli fractional differential equation}

General form of Bernoulli fractional differential equations is as follows,

$T_{\alpha}(u)(x)+P(\mathrm{x}) u(x)=Q(x)(u(x))^{n}$,

Where $\alpha$-differentiable functions and $u(x)$ is an unknown function.

Using property $(\mathrm{F})$ in equation (4), reads

$x^{1-\alpha} u^{\prime}(x)+P(x) u(x)=Q(x)(u(x))^{n}$,

$u^{\prime}(x)+\frac{P(x)}{x^{1-\alpha}} u(x)=\frac{Q(x)}{x^{1-\alpha}}(u(x))^{n}$,

Where equation (10) is the Bernoulli equation (see [15]). We know that it will be linear for $n=0$ or 1 , it can be reduced to a linear ordinary equation for any other value of $n$ by the change of dependent variable $z=(u(x))^{1-n}$, as a result, according to the results in section (3) the general solution is as follows,

$(u(x))^{1-n}=e^{-\int(1-n) \frac{P(x)}{x^{1-\alpha}} d x}\left(\int(1-n) \frac{Q(x)}{x^{1-\alpha}} e^{\int(1-n) \frac{P(x)}{x^{1-\alpha}} d x} d x+C\right)$

Consequently, by using subsection (2.2) and mentioned procedure to equation (11), the general solution of equation (9) is as the following

$u(x)=\left(e^{-I_{\alpha}((1-n) P(x))}\left[I_{\alpha}\left((1-n) Q(x) \cdot e^{I_{\alpha}((1-n) P(x))}\right)+C\right]\right)^{\frac{1}{1-n}}$.

Example 3 According to (12), a general solution to the following Bernoulli fractional differential equations, based on conformable fractional derivative

$T_{\frac{1}{2}}(u)(x)+u(x)=\left(x^{2}-x \sqrt{x}\right)(u(x))^{2}$,

Can be presented as the following

$u(x)=\left(x^{2}+C e^{2 \sqrt{x}}\right)^{-1}$.

Example 4 According to equation (12) the Bernoulli fractional differential equation

$T_{\frac{1}{2}}(u)(x)+\sqrt{x} u(x)=\left(x e^{-2 x}\right)(u(x))^{-1}$,

Has the following general solution

$u(x)=\sqrt{e^{-2 x}\left(\frac{4}{3} x \sqrt{x}+C\right)}$.

\subsection{Riccati fractional differential equation}

A natural extension of a first order fractional differential equation is the Riccati fractional differential equations,

$T_{\alpha}(u(x))=P(x)+Q(x) u(x)+R(x)(u(x))^{2}$,

Where $\mathrm{P}(x), Q(x), R(x)$ are $\alpha$-differentiable functions, and $u(x)$ is an unknown function.

If a particular solution $u_{1}(x)$ is known, then general solution has the form $u(x)=u_{1}(x)+z(x)$ where $z(x)$ is a general solution of the following Bernoulli fractional differential equation,

$$
T_{\alpha}(z(x))+\left(-Q(x)-2 R(x) u_{1}(x)\right) z(x)=R(x)(z(x))^{2} .
$$

Example 5 Find the general solution of the Riccati fractional differential equations, 


$$
T_{\frac{1}{2}}(u(x))=-x \sqrt{x}+\frac{1}{2 \sqrt{x}} u(x)+\sqrt{x}(u(x))^{2},
$$

Since $u_{1}(x)=\sqrt{x}$ is an obvious particular solution.

To obtain a general solution to Riccati equation, suppose $u(x)=$ $u_{1}(x)+z(x)$, where the function $z(x)$ is denoted by $z(x)=$ $\frac{2 \sqrt{x} e^{\frac{4}{3} x \sqrt{x}}}{C-e^{\frac{4}{3} x \sqrt{x}}}$, so a general solution of equation is as the following

$u(x)=\sqrt{x}+\frac{2 \sqrt{x} e^{\frac{4}{3} x \sqrt{x}}}{C-e^{\frac{4}{3} x \sqrt{x}}}$

Example 6 Find the general solution of the following Riccati fractional differential equations,

$T_{\frac{1}{2}}(u(x))=-2 x^{2} \sqrt{x}+\frac{1}{\sqrt{x}} u(x)+2 \sqrt{x}(u(x))^{2}$,

Which has $u_{1}(x)=x$, as an obvious particular solution.

Suppose $u(x)=u_{1}(x)+z(x)$ where the function $z(x)$ is denoted by $(x)=\frac{4 x e^{2 x^{2}}}{C-2 e^{2 x^{2}}}$, consequently a general solution of the Riccati fractional differential equations is as the following

$u(x)=x+\frac{4 x e^{2 x^{2}}}{C-2 e^{2 x^{2}}}$

\section{Conclusion}

In this article, Bernoulli and Riccati fractional different equations have been solved regarding the general solution of first order linear fractional differential equations, based on conformable fractional derivative. This approach lead to the exact solution, so there was no need for using any numerical approach.

\section{References}

[1] R. Khalil, M. A. Horani, A. Yousef and M. Sababheh, A new definition of fractional derivative, J. Comput. Appl. Math., 264, 65-70 (2014). https://doi.org/10.1016/j.cam.2014.01.002.

[2] T. Abdeljawad, on conformable fractional calculus, Journal of Computational and Applied Mathematics., 279, 57-66 (2015). https://doi.org/10.1016/j.cam.2014.10.016.

[3] V. Daftardar -Gejji, H. Jafari, Solving a multi- order fractional differential equation using Adomian Decomposition, Appl. Math. Comput., 189 (2007) 541-548. https://doi.org/10.1016/j.amc.2006.11.129.

[4] B. Ghazanfari, A. Sepahvandzadeh, Adomian Decomposition Method for solving Fractional Bratu-type equations, J. Math. Computer and Science, 8 (2014) 236-244.

[5] O. Abdulaziz, I. Hashim, S. Momani, solving systems of fractional differential equations by homotopy perturbation method, Phys. Let., A 372 (2008) 451-459.

[6] B. Ghazanfari, A. G. Ghazanfari, M. Fuladvand, Modification of the Homotopy Perturbation Method for numerical solution of Nonlinear Wave and of Nonlinear Wave Equations., J. Math. Computer Sci. 3 (2011) 212-224.

[7] M. Rabbani, New Homotopy Perturbation Method to Solve Non-Linear Problems, J. Math. Computer Sci., 7 (20013) 272-275.

[8] I. Hashim, O. Abdulaziz, S. Momani, Homotopy Analysis Method for fractional IVPs, Commun. Nonlinear Sci. Numer. Simul. 14 (2009) 674-684. https://doi.org/10.1016/j.cnsns.2007.09.014.

[9] G. Wu, E. W. M. Lee, Fractional variational iteration method and its application, Phys. Lett., A374 (2010) 2506-2509. https://doi.org/10.1016/j.physleta.2010.04.034.

[10] Z. Odibat, S. Momani, V. Suat Erturk, Generalized differential transform method: application to differential equations of fractional order, Appl. Math. Comput., $197 \quad$ (2008) 467-477. https://doi.org/10.1016/j.amc.2007.07.068.

[11] Y. Zhang, A finite difference method for fractional partial differential equation, Appl. Math. Comput, 215 (2009) 524-529. https://doi.org/10.1016/j.amc.2009.05.018.

[12] Hossein. Aminikhah, Amir Hossein Refahi Sheikhani, Hadi Rezazadeh. Approximate analytical solution of distributed order fractional Riccati differential equation, Ain Shams Eng J (2016).
[13] H. Aminikhah, A. H. Refahi sheikhani, H. Rezazadeh, Sub-equation method for the fractional regularized long-wave equations whit conformable fractional derivatives, Scientia Iranica B (2016) 23(3), 1048-1054.

[14] Bothayna S.H. Kashkari, Muhammed I. Syam, Fractional-order Legendre operational matrix of fractional integration for solving the Riccati equation with fractional order, Applied Mathematics and $\begin{array}{lll}\text { Computation } & 290 & \text { (2016) 281-291 }\end{array}$ https://doi.org/10.1016/j.amc.2016.06.003.

[15] George F. Simmons, Differential Equations Whit Applications And Historical Notes, McGraw-Hill,Inc. New York. 1974. 